\title{
The homologous regulators ANR of Pseudomonas aeruginosa and FNR of Escherichia coli have overlapping but distinct specificities for anaerobically inducible promoters
}

\author{
Harald V. Winteler and Dieter Haas
}

Laboratoire de Biologie Microbienne, Université de Lausanne, $\mathrm{CH}-1015$ Lausanne, Switzerland
Author for correspondence: Dieter Haas. Tel: +412169256 31. Fax: +41216925635.

The anaerobic transcriptional regulator ANR induces the arginine deiminase and denitrification pathways in Pseudomonas aeruginosa during oxygen limitation. The homologous activator FNR of Escherichia coli, when introduced into an anr mutant of $P$. aeruginosa, could functionally replace ANR for anaerobic growth on nitrate but not for anaerobic induction of arginine deiminase. In an FNR-positive E. coli strain, the ANR-dependent promoter of the arcDABC operon, which encodes the enzymes of the arginine deiminase pathway, was not expressed. To analyse systematically these distinct induction patterns, a lacZ promoter-probe, broad-host-range plasmid containing various -40 regions (the ANR/FNR recognition sequences) and - 10 promoter sequences was constructed. These constructs were tested in $P$. aeruginosa and in E. coli expressing either ANR or FNR. In conjunction with the consensus - 10 hexamer of $E$. coli $\sigma^{70}$ RNA polymerase (TATAAT), the consensus FNR site (TTGAT ....ATCAA) was recognized efficiently by ANR and FNR in both hosts. By contrast, when promoters contained the Arc box (TTGAC.... ATCAG), which is found in the arcDABC promoter, or a symmetrical mutant FNR site (CTGAT.... ATCAG), ANR was a more effective activator than was FNR. Conversely, an extended 22 bp, fully symmetrical FNR site allowed better activation with FNR than with ANR. Combination of the arc promoter - 10 sequence (CCTAAT) with the Arc box or the consensus FNR site resulted in good ANR-dependent expression in $P$. aeruginosa but gave practically no expression in E. coli, suggesting that RNA polymerase of $P$. aeruginosa differs from the $E$. coli enzyme in -10 recognition specificity. In conclusion, ANR and FNR are able to activate the RNA polymerases of $P$. aeruginosa and $E$. coli when the -40 and -10 promoter elements are identical or close to the $E$. coli consensus sequences.

Keywords: Pseudomonas aeruginosa, anaerobic control, ANR, FNR, arcD $A B C$ operon

\section{INTRODUCTION}

The anr gene of Pseudomonas aeruginosa is required for the expression of physiological functions which are inducible under oxygen-limiting or anaerobic conditions: the arginine deiminase pathway, denitrification and cyanogenesis (Galimand et al., 1991; Zimmermann et al., 1991;

Abbreviations: Ap, ampicillin (resistance); $\mathrm{Cb}$, carbenicillin (resistance); $\mathrm{Km}$, kanamycin (resistance); Tc, tetracycline (resistance).
Sawers, 1991; Haas et al., 1992; Arai et al., 1995a; Ye et al., 1995). The anr gene product is a protein which has $51 \%$ amino acid sequence identity with the anaerobic transcriptional regulator FNR of Escherichia coli (Sawers, 1991; Zimmermann et al., 1991). Several promoters of $P$. aeruginosa are positively controlled by ANR (Haas $e t$ al., 1992; Arai et al., 1995a); of these, the promoter of the $\operatorname{arc} D A B C$ operon has been investigated in most detail (Galimand et al., 1991; Gamper et al., 1991). The arc operon encodes the enzymes of the arginine deiminase 
pathway and thus enables $P$. aeruginosa to grow anaerobically with arginine as the sole energy source (Lüthi et al., 1990). The -40 region of the arc promoter contains a sequence termed the Arc box, TTGAC.... ATCAG, which is closely related to the consensus FNRbinding sequence TTGAT.... ATCAA in E. coli (Spiro \& Guest, 1990; Unden et al., 1995). Mutational analysis of the arc promoter has shown that the Arc box is essential for ANR-dependent induction of the arc operon under oxygen-limiting conditions and that the -10 hexamer CCTAAT is also important for transcription of the arc genes. By contrast, the -35 region does not appear to be critical (Gamper et al., 1991).

Transcriptional regulators of the FNR family occur in many Gram-negative and Gram-positive bacteria. Some members of this family, including FNR and ANR, are active under oxygen-limiting conditions; these regulators typically contain four essential and conserved cysteine residues, three of which lie in the N-terminal part of the protein (Green et al., 1993; Spiro, 1994). It has been proposed that $\mathrm{Fe}^{2+}$ may be bound to the conserved cysteines in the active form of FNR and that oxidation of $\mathrm{Fe}^{2+}$ in the presence of $\mathrm{O}_{2}$ may inactivate $\mathrm{FNR}$ (Unden $e t$ al., 1995). However, the recent discovery of an iron-sulfur centre in FNR indicates that the mechanism of sensing the redox state may be more complex (Khoroshilova $e t$ al., 1995). Some proteins of the FNR family, for instance DNR, do not have the conserved cysteine residues; DNR is a transcriptional regulator which is needed, in addition to ANR, for denitrification in $P$. aeruginosa (Arai et al., 1995b).

A helix-turn-helix motif located in the C-terminal part of FNR is involved in the binding of the regulator to the conserved -40 region, as demonstrated by the effects of specific mutational changes in FNR (Spiro \& Guest, 1987; Spiro et al., 1990; Lazazzera et al., 1993) and by footprinting and bandshift experiments (Green et al., 1991; Sharrocks et al., 1991; Green \& Guest, 1994; Ziegelhoffer \& Kiley, 1995). From these studies it is concluded that the FNR residues Glu-209, Ser-212 and Arg-213 in the second helix of the helix-turn-helix motif have a significant role in the recognition of the FNR box. The same residues are conserved in ANR (Zimmermann et al., 1991). The FNR homologues of non-enteric bacteria all interact with -40 promoter regions having a TTGAT .... ATCAA consensus (i.e. an FNR box) (Spiro, 1994). It has been verified experimentally that this consensus sequence is recognized by ANR (Lodge et al., 1990; Galimand et al., 1991).

The cloned intact $\operatorname{arcD} A B C$ operon of $P$. aeruginosa is expressed at very low, uninduced levels in $\mathrm{Fnr}^{+} E$. coli strains (Lüthi et al., 1986; Galimand et al., 1991). Strong $E$. coli promoters fused to the arc operon allow good expression of the arginine deiminase pathway enzymes in E. coli, indicating that the arc promoter rather than other features (such as codon usage) accounts for the poor expression of the arc operon in the heterologous host (Lüthi et al., 1986; Gamper et al., 1991). Low activity of the arc promoter in E. coli may be a consequence of poor recognition of the Arc box by FNR and/or poor interaction between the E. coli RNA polymerase and the -10 hexamer CCTAAT of the arc promoter. Using synthetic, modular promoter constructs, we show here that both factors are important.

\section{METHODS}

Bacterial strains and growth conditions. The $P$. aeruginosa strains used were the wild-type PAO1 and its anr mutant derivative PAO6261 (Ye et al., 1995). The construction of strain PAO6265, a PAO1 derivative carrying a lac $Z$ transcriptional fusion in the chromosomal arcD gene, will be described elsewhere. The E. coli strains were MC1000 [ $\Delta($ lacIZY $A) X 74$ galU galK $\Delta$ (ara-leu) rpsL; Eiglmeier et al., 1989] and its mutant derivative JRG1728 [ $\Delta(t y r R-f n r-t r g)$; Spiro \& Guest, 1987].

All incubations were carried out at $37^{\circ} \mathrm{C}$. $P$. aeruginosa was grown in yeast extract arginine medium under aerobic and oxygen-limiting conditions as described by Galimand et al. (1991). Anaerobic growth was tested on nutrient agar supplemented with $0.2 \% \mathrm{KNO}_{3}$ in an anaerobic jar. Plasmid maintenance was ensured by the addition of $100 \mu \mathrm{g}$ carbenicillin $\mathrm{ml}^{-1}$ or $125 \mu \mathrm{g}$ tetracycline $\mathrm{ml}^{-1}$. E. coli was grown in $60 \mathrm{ml}$ Luria broth containing $0.5 \%$ glucose (Sambrook et al., 1989), either aerobically with vigorous shaking in a baffled $500 \mathrm{ml}$ flask or under oxygen limitation with gentle shaking in a hermetically sealed $125 \mathrm{ml}$ bottle. Plasmid maintenance was ensured by the addition of $100 \mu \mathrm{g}$ ampicillin $\mathrm{ml}^{-1}$ or $50 \mu \mathrm{g}$ kanamycin $\mathrm{ml}^{-1}$. Both $P$. aeruginosa and $E$. coli cells were harvested in the early exponential phase $\left(<5 \times 10^{8}\right.$ cells $\left.\mathrm{ml}^{-1}\right)$ for aerobic incubations and in early stationary phase (approximately $8 \times 10^{8}$ cells ml $^{-1}$ ) for oxygen-limiting conditions.

DNA manipulation and plasmid constructions. Plasmid DNA was isolated by the method of Del Sal et al. (1988) and sequenced by the chain-termination method with Sequenase (US Biochemical) according to the manufacturer's instructions. Plasmid pRK2501 fnr (Tc, RK2 replicon, $11.7 \mathrm{~kb}$ ), which carries the $E$. coli fnr gene on a $1.64 \mathrm{~kb} \mathrm{BamHI}-$ HindIII fragment (Shaw \& Guest, 1982) in pRK2501 (Kahn et al., 1979), was kindly supplied by S. Busby, University of Birmingham, UK. Plasmid pME3777 (Km, ColE1 replicon, $7.8 \mathrm{~kb}$ ) carries the $P$. aeruginosa anr gene downstream of the tac promoter and is a $\mathrm{Km}$-resistant derivative of pME3587 (Zimmermann et al., 1991); a 1.2 kb Km cassette (the SmaI fragment of pUC4-KIXX; Pharmacia) was inserted into the ScaI site of pME3587. The modular plasmid pME3533 (Fig. 1) and its derivatives were constructed as follows. Three sets of complementary oligonucleotides ('modules') were synthesized: a 53-mer/61-mer pair with a 5' PstI site and a $3^{\prime}$ HindIII site carrying a fragment of the $E$. coli lac $Z$ sequence ( +1 transcriptional start site, ribosome-binding site and the first six codons of lac $Z$ ) (Dreyfus, 1988; Minton, 1984), two different 28 -mer/20-mer pairs with a $5^{\prime}$ Sall site and a $3^{\prime}$ PstI site ( -10 hexamers) and six different 28 -mer/28-mer pairs with a $5^{\prime}$ Bam HI site and a $3^{\prime}$ Sall site ( -40 boxes). One Bam HI-SalI module, one SalI-PstI module and the PstIHindIII module were ligated together in the vector pNM480 (Ap/Cb, ColE1 replicon, $8.6 \mathrm{~kb}$; Minton, 1984), a translational lac $Z$ fusion vector. The $3.2 \mathrm{~kb} \mathrm{Bam} \mathrm{HI} / A b a I I I$ fragment carrying the $l a c Z$ gene and various modules was taken from the pNM480 derivatives and cloned into the broad-host-range vector pKT240 (Bagdasarian et al., 1983) cut with BamHI and HpaI, resulting in the plasmids pME3533, pME3534, pME3547, pME3548, pME3549, pME3769, pME3770, pME3771, pME3773, pME3780 and pME3781 (Ap/Cb lac $Z^{+}$Mob IncQ, $13.5 \mathrm{~kb}$; Fig. 1, Tables 1-4). All promoter constructs were 
verified by nucleotide sequencing. The plasmids were introduced into $P$. aeruginosa by electroporation as described by Zimmermann et al. (1991).

Enzyme assay. $\beta$-Galactosidase specific activities were measured in toluenized cells and are expressed in thousands of Miller units (kU) (Sambrook et al., 1989). All enzyme assay data are mean values from at least three independent experiments. The measurements generally differed by $<20 \%$. Arginine deiminase was assayed as described previously (Lüthi et al., 1986).

\section{RESULTS}

\section{Design of artificial modular promoters which depend on ANR/FNR activation}

To study the functions of anaerobically inducible promoters in $P$. aeruginosa and $E$. coli, we constructed a series of synthetic promoters. Different -40 regions including the consensus FNR-binding site, the Arc box and various mutant derivatives were combined, via a SalI site, with either the arc -10 hexamer CCTAAT or the consensus -10 hexamer TATAAT of $E$. coli $\sigma^{70}$ RNA polymerase (Fig. 1). These artificial promoters, which lack a typical -35 sequence, were inserted upstream of the $E$. coli lac $Z$ gene with its own ribosome-binding site, in a broad-host-range IncQ vector plasmid. The prototype plasmid pME3533 (Fig. 1; Table 1) represents the wildtype arc promoter situation, except for the base changes introduced to create restriction sites (BamHI, SalI and PstI) flanking the modules. In a previous study we have shown that the expression of the arc operon remains unchanged when the arc promoter region lying between positions -34 and -19 is substituted by a linker which is different from the arc wild-type sequence at every base, but without altering the spacing between the -40 and the -10 regions (Gamper $e t$ al., 1991). Thus, the artificial SalI site (Fig. 1) is not likely to affect promoter activity.

Plasmid pME3781 (Fig. 1) contains the consensus FNR site and the consensus -10 hexamer. The different $-40 /-10$ combinations are listed in Tables 1-4. A plasmid containing only the lac $Z$ reporter gene, pME3536 (Fig. 1), gave negligible background $\beta$-galactosidase activities (Table 1) and may be useful as a promoter-probe system.

\section{ANR, in contrast to FNR, recognizes the Arc box efficiently in $P$. aeruginosa}

A first series of -40 regions was tested in combination with the arc -10 hexamer CCTAAT in $P$. aeruginosa as the host (Table 1). A strain having a deletion in the anr gene, PAO6261, gave low, virtually uninduced background values of $\beta$-galactosidase activity (Table 1 ). The ANRpositive wild-type strain PAO1 expressed high $\beta$ galactosidase levels which were inducible about 10 -fold by oxygen limitation, when the -40 region corresponded to the consensus FNR site TTGAT .... ATCAA or to the Arc box TTGAC .... ATCAG (Table 1). This experiment confirms that ANR activates the artificial promoters used. Moreover, deletion of the first half-site of the Arc box (in pME3533-1) abolished induction, in agreement with earlier results (Gamper et al., 1991). The fact that the Arc box resulted in a $\beta$-galactosidase level that was about $30 \%$ below that specified by the FNR box (Table 1) was essentially due to the $G$ at the 5 th position of the second half-site rather than to the $C$ at the 5 th position of the first half-site (Table 1). The symmetrical -40 sequence CTGAT .... ATCAG gave low, but detectable ANRdependent induction (Table 1).

The anr-negative mutant PAO6261 (Ye et al., 1995) does not grow anaerobically on nitrate nor does it utilize arginine as an anaerobic energy source via the arginine deiminase pathway. The $E$. coli fnr gene was introduced into this mutant on an IncP replicon (pRK2501 fnr). FNR restored anaerobic growth of strain PAO6261 on nitrate but failed to induce the chromosomal arc operon: under oxygen-limiting conditions, arginine deiminase had a specific activity of $65 \mathrm{U} \mathrm{mg}^{-1}$ in the wild-type PAO1 but remained at the uninduced level of $2.3 \mathrm{U} \mathrm{mg}^{-1}$ in strain PAO6261/pRK2501 fnr.

The promoter constructs were then tested in strain PAO6261 expressing FNR (Table 1). The consensus FNR site TTGAT.... ATCAA allowed good induction by oxygen depletion. Although the induced $\beta$-galactosidase level was lower than that seen in the presence of ANR, it is clear that FNR of E. coli and RNA polymerase of $P$. aeruginosa do interact positively with this type of promoter. By contrast, when the promoter contained the Arc box TTGAC .... ATCAG, activation by FNR was abolished almost totally (Table 1); this fact explains why FNR could not induce the chromosomal arc operon. FNR tolerated a $\mathrm{T} \rightarrow \mathrm{C}$ change at the fifth position of the first half-site in the FNR box but gave very poor induction when the 5 th position of the second half-site was changed from $A$ to $G$ (Table 1). The symmetrical construct CTGAT.... ATCAG was not recognized by FNR in $P$. aeruginosa (Table 1), corroborating earlier data obtained in E. coli (Lodge et al., 1990). The results indicate that ANR and FNR differ with respect to recognition specificity, the base at the 5 th position of the second half-site being especially important.

\section{The consensus -10 hexamer TATAAT enhances aerobic and anaerobic expression in $P$. aeruginosa}

The -40 regions described above were combined with the $E$. coli consensus -10 hexamer TATAAT instead of CCTAAT, and the expression of the lac $Z$ reporter gene was again determined in $P$. aeruginosa. This change of the -10 sequence resulted in a three- to fourfold increase of both the aerobic and anaerobic expression levels and led to relatively high background activities in the anr mutant PAO6261 (Table 2). However, if we subtract these background activities from the values obtained in the presence of ANR (in strain PAO1) or FNR (in strain PAO6261/pRK2501 fnr), then the differential activation abilities of ANR and FNR remain essentially unchanged. In particular, the Arc box allowed high expression with ANR under oxygen-limiting conditions whereas expression was much lower with FNR (Table 2). A control plasmid containing a very poorly conserved FNR 

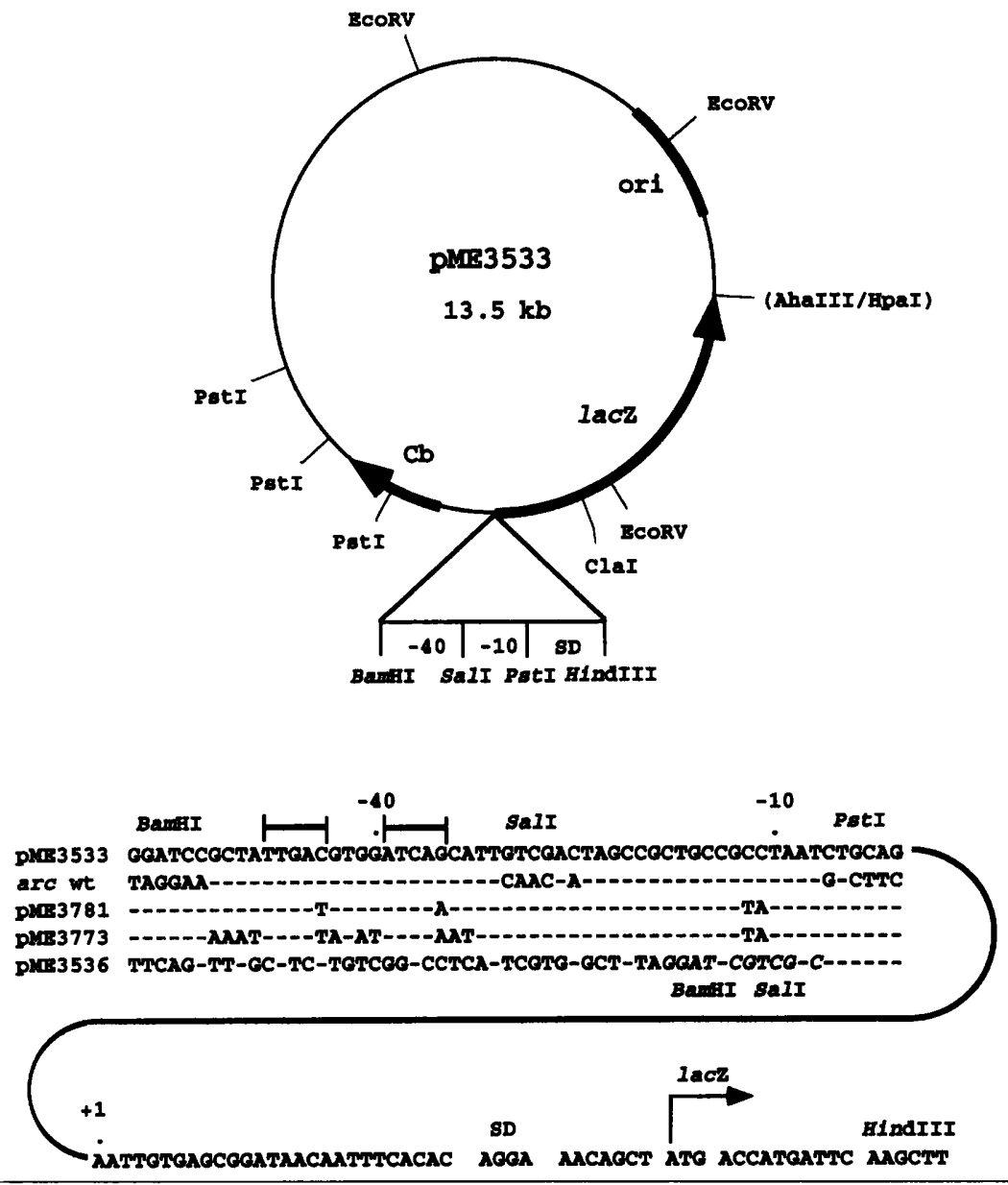

Fig. 1. Construction of artificial modular promoters with a lacZ reporter gene in the broad-host-range IncQ vector PKT240. The promoter modules are derived from the wild-type arc promoter. They were assembled as described in Methods. Four constructs are shown as examples. Plasmid pME3533 corresponds exactly to the arc promoter (shown below) in the -40 and the -10 regions. Plasmid PME3781 carries the $E$. coli consensus FNR inner motif and the consensus -10 hexamer, and plasmid pME3773 carries the extended 22 bp consensus FNR-binding site upstream of the consensus -10 sequence. Plasmid pME3536 does not contain the $-40 /-10$ promoter modules and therefore may serve as a promoter-probe vector with broad-hostrange properties, using the unique BamHI and Sall sites. The last line shows the transcriptional and translational starts of the $E$. coli lacZ gene including its own Shine-Dalgarno (SD) sequence and the first six lac $Z$ codons. The various $-\mathbf{4 0}$ modules are given in Tables 1-4. Restriction sites lost after cloning are indicated in parentheses.

Table 1. Differential recognition of the -40 promoter region, combined with the suboptimal -10 hexamer CCTAAT, by ANR and FNR in $P$. aeruginosa

Deviations from the consensus FNR box are indicated by bold letters.

\begin{tabular}{|c|c|c|c|c|c|c|c|}
\hline \multirow[t]{3}{*}{ Plasmid } & \multirow[t]{3}{*}{-40 Promoter region } & \multicolumn{6}{|c|}{$\beta$-Galactosidase specific activity $(\mathrm{kU}) *$} \\
\hline & & \multicolumn{2}{|c|}{$\begin{array}{l}\text { PAO1 } \\
\left(a n r^{+}\right)\end{array}$} & \multicolumn{2}{|c|}{$\begin{array}{c}\text { PAO6261/ } \\
\text { PRK2501-fnr } \\
\left(f n r^{+}\right)\end{array}$} & \multicolumn{2}{|c|}{$\begin{array}{c}\text { PAO6261 } \\
\text { (anr) }\end{array}$} \\
\hline & & $+\mathbf{O}_{2}$ & $-\mathbf{O}_{2}$ & $+\mathbf{O}_{2}$ & $-\mathbf{O}_{2}$ & $+\mathrm{O}_{2}$ & $-\mathrm{O}_{2}$ \\
\hline pME3780 & TTGAT .... ATCAA & $4 \cdot 1$ & $37 \cdot 2$ & 0.8 & $11 \cdot 7$ & 0.5 & 0.7 \\
\hline pME3533 & TTGAC .... ATCAG & $2 \cdot 4$ & $24 \cdot 5$ & 0.7 & $2 \cdot 5$ & 0.6 & 0.9 \\
\hline pME3547 & TTGAC.... ATCAA & $2 \cdot 6$ & $35 \cdot 5$ & $1 \cdot 2$ & $10 \cdot 9$ & $0 \cdot 4$ & 0.6 \\
\hline pME3549 & TTGAT .... ATCAG & $2 \cdot 0$ & $27 \cdot 2$ & $1 \cdot 0$ & 3.8 & ND & ND \\
\hline pME3770 & CTGAT .... ATCAG & $0 \cdot 6$ & $4 \cdot 5$ & $1 \cdot 8$ & $2 \cdot 9$ & $1 \cdot 2$ & $1 \cdot 8$ \\
\hline pME3533-1† & CGCTC .... ATCAG & 0.5 & $0 \cdot 3$ & ND & ND & ND & ND \\
\hline pME3536 & CTTCC.... GGCCC & $0 \cdot 2$ & $0 \cdot 3$ & ND & ND & $0 \cdot 1$ & $0 \cdot 3$ \\
\hline
\end{tabular}

* $\beta$-Galactosidase activities are expressed in thousands of Miller units $(\mathrm{kU})$. Values obtained in strain PAO6261 without plasmid are the means of two experiments. All other measurements were carried out at least three times. $+\mathrm{O}_{2}$, aerobic growth conditions; $-\mathrm{O}_{2}$, oxygen-limiting growth conditions. ND, Not determined.

tpME3533-1 carries a spontaneous $17 \mathrm{bp}$ deletion encompassing the first half-site of the ANR box in pME3533. 
Table 2. Differential recognition of the -40 promoter region, combined with the consensus -10 hexamer TATAAT, by ANR and FNR in $P$. aeruginosa

Deviations from the consensus FNR box are indicated by bold letters. See first footnote of Table 1 for further details.

\begin{tabular}{|c|c|c|c|c|c|c|c|}
\hline \multirow[t]{3}{*}{ Plasmid } & \multirow[t]{3}{*}{-40 Promoter region } & \multicolumn{6}{|c|}{$\beta$-Galactosidase specific activity (kU) } \\
\hline & & \multicolumn{2}{|c|}{$\begin{array}{l}\text { PAO1 } \\
\left(\text { anr }^{+}\right)\end{array}$} & \multicolumn{2}{|c|}{$\begin{array}{l}\text { PAO6261/ } \\
\text { PRK2501-fnr } \\
\left(f n r^{+}\right)\end{array}$} & \multicolumn{2}{|c|}{$\begin{array}{c}\text { PAO6261 } \\
\text { (anr) }\end{array}$} \\
\hline & & $+\mathbf{O}_{2}$ & $-\mathbf{O}_{2}$ & $+O_{2}$ & $-\mathbf{O}_{2}$ & $+\mathbf{O}_{2}$ & $-\mathbf{O}_{2}$ \\
\hline pME3781 & TTGAT .... ATCAA & $16 \cdot 2$ & $89 \cdot 0$ & $4 \cdot 9$ & $41 \cdot 0$ & $2 \cdot 5$ & $5 \cdot 1$ \\
\hline pME3534 & TTGAC .... ATCAG & $11 \cdot 2$ & $75 \cdot 0$ & $2 \cdot 0$ & $15 \cdot 6$ & 1.9 & $3 \cdot 8$ \\
\hline pME3548 & TTGAC .... ATCAA & $11 \cdot 6$ & $79 \cdot 1$ & $5 \cdot 0$ & $32 \cdot 4$ & $2 \cdot 4$ & $5 \cdot 6$ \\
\hline pME3769 & TTGAT .... ATCAG & $11 \cdot 0$ & $80 \cdot 9$ & $2 \cdot 6$ & $14 \cdot 7$ & $1 \cdot 4$ & $3 \cdot 5$ \\
\hline pME3771 & CTGAT .... ATCAG & $6 \cdot 2$ & $35 \cdot 1$ & $2 \cdot 9$ & $10 \cdot 8$ & $2 \cdot 4$ & $4 \cdot 0$ \\
\hline pME3771-1* & ACTGA .... GACAG & $5 \cdot 2$ & 8.9 & $7 \cdot 7$ & 8.7 & 6.8 & 5.9 \\
\hline
\end{tabular}

* This construct is a spontaneous deletion derivative of pME3771: the T in the second half-site of the ANR box was deleted.

box (pME3771-1) showed no ANR- or FNR-dependent induction (Table 2), as expected.

In induced $P$. aeruginosa cells containing an ANRresponsive promoter (e.g. on pME3781), $\beta$-galactosidase represented at least $20 \%$ of the total cellular protein (data not shown). This high expression was due, in part, to the fact that the IncQ vector used has about 30 copies in $P$. aeruginosa (Jeenes et al., 1986). It could be argued that under these conditions the amount of ANR present in the cell might be sub-optimal and thus limit the expression. Therefore, we tested whether an increase of the anr copy number would enhance $\beta$-galactosidase expression. However, when the anr gene was introduced on the IncP plasmid pME3552 (Galimand et al., 1991) into $P$. aeruginosa, the $\beta$-galactosidase levels specified by pME3781 did not change (data not shown).

In conclusion, the $E$. coli consensus -10 sequence TATAAT resulted in high aerobic and anaerobic expression in $P$. aeruginosa. In E. coli similar effects have been observed: the FNR-dependent narGHJI promoter shows high aerobic and anaerobic expression with a consensus -10 sequence (Walker \& DeMoss 1991, 1992).

\section{$E$. coli does not recognize the $P$. aeruginosa arc promoter}

Two -40 regions (Arc box, FNR consensus) with the arc wild-type -10 hexamer and five -40 regions with the E. coli consensus -10 hexamer were also tested in E. coli. The effect of FNR was measured in strain MC1000 $\left(\mathrm{frr}^{+}\right)$ and that of ANR was assessed in strain JRG1728 (fnr) carrying the anr gene in pME3777 (Table 3). The fnr-negative host JRG1728 gave negligible, uninduced $\beta$-galactosidase levels with all constructs tested (data not shown). From previous work (Galimand et al., 1991; Sawers, 1991;
Zimmermann et al., 1991) it is known that ANR can be expressed in E. coli and functionally replace FNR at some promoters. The native anr promoter is practically inactive in $E$. coli, giving $\leqslant 2 \%$ of the expression found in $P$. aeruginosa (Sawers, 1991; Savioz et al., 1993). Therefore, in pME3777 the anr gene was expressed from the tac promoter, which needed to be induced with IPTG in order to achieve complete activation of the anaerobically inducible promoters (data not shown).

The plasmid constructs containing the CCTAAT hexamer were expressed very poorly or not at all in $E$. coli; the slight FNR-dependent induction with the FNR consensus box was at the limit of detection in our routine $\beta$ galactosidase assays (Table 3 ). Exchanging CCTAAT with the consensus TATAAT led to high and regulated expression in $E$. coli. This indicates that the native $P$. aeruginosa -10 sequence constitutes a major barrier to expression of the arc operon in E. coli.

With TATAAT as the -10 hexamer, ANR activated promoters carrying the Arc box, the consensus FNR site or the single half-site mutant boxes (in pME3548 and pME3769) with similar efficiencies of $80-110 \%$, the anaerobic expression provided by the consensus FNR site being defined as $100 \%$ (Table 3 ). These results are in good agreement with the parallel data obtained in $P$. aeruginosa, where the efficiencies of anaerobic expression varied between 85 and $100 \%$ (Table 2). Recognition of the symmetrical CTGAT.... ATCAG mutant -40 sequence by ANR appeared to be good in E. coli $(80 \%$ efficiency) and superior to that in $P$. aeruginosa (37\% efficiency; Tables 2 and 3), for reasons that are not obvious.

FNR effectively activated the promoters containing the consensus FNR site or the TTGAC half-site mutant 
Table 3. Differential recognition of the -40 promoter region by ANR and FNR in $E$. coli

Deviations from the consensus FNR box are indicated by bold letters. See first footnote of Table 1 for further details.

\begin{tabular}{|c|c|c|c|c|c|c|}
\hline \multirow[t]{3}{*}{ Plasmid } & \multirow{3}{*}{$\begin{array}{c}-40 \\
\text { Region }\end{array}$} & \multirow{3}{*}{$\begin{array}{c}-10 \\
\text { Region }\end{array}$} & \multicolumn{4}{|c|}{$\beta$-Galactosidase specific activity $(\mathrm{kU})$} \\
\hline & & & \multicolumn{2}{|c|}{$\begin{array}{c}\text { JRG1728/ } \\
\text { pME3777 } \\
\left.\text { (anr }^{+}\right)^{*}\end{array}$} & \multicolumn{2}{|c|}{$\begin{array}{c}\text { MC1000 } \\
\left(f n r^{+}\right)\end{array}$} \\
\hline & & & $+\mathrm{O}_{2}$ & $-\mathbf{O}_{2}$ & $+\mathrm{O}_{2}$ & $-\mathbf{O}_{2}$ \\
\hline pME3780 & TTGAT .... ATCAA & CCTAAT & $<0.01$ & $<0.01$ & 0.01 & $0 \cdot 04$ \\
\hline pME3533 & TTGAC .... ATCAG & CCTAAT & $<0.01$ & $<0.01$ & $<0.01$ & $<0.01$ \\
\hline pME3781 & TTGAT .... ATCAA & TATAAT & $3 \cdot 3$ & $20 \cdot 1$ & $3 \cdot 9$ & $31 \cdot 8$ \\
\hline pME3534 & TTGAC .... ATCAG & TATAAT & 1.7 & $17 \cdot 3$ & $2 \cdot 4$ & $13 \cdot 1$ \\
\hline pME3548 & TTGAC .... ATCAA & TATAAT & $3 \cdot 3$ & $22 \cdot 2$ & 3.6 & $28 \cdot 6$ \\
\hline pME3769 & TTGAT .... ATCAG & TATAAT & 1.5 & $16 \cdot 3$ & $2 \cdot 0$ & $14 \cdot 4$ \\
\hline pME3771 & CTGAT .... ATCAG & TATAAT & 0.9 & $16 \cdot 1$ & 0.5 & $2 \cdot 0$ \\
\hline
\end{tabular}

* The fnr mutant JRG1728 carries the anr gene of $P$. aeruginosa downstream of the tac promoter on pME3777 (see Methods). IPTG (1 mM) was used to induce the tac promoter.

Table 4. Differential recognition of -40 promoter regions, combined with the consensus -10 hexamer TATAAT, by ANR and FNR in $P$. aeruginosa and $E$. coli

Bold letters indicate the consensus FNR site as defined elsewhere in this study. The extended, symmetrical consensus FNR site of pME3773 has been proposed by Bell et al. (1989).

\begin{tabular}{|c|c|c|c|c|}
\hline \multirow[t]{3}{*}{ Plasmid } & \multirow[t]{3}{*}{-40 Promoter region } & \multicolumn{3}{|c|}{ Relative specific activity of LacZ reporter (\%)* } \\
\hline & & \multicolumn{2}{|c|}{ ANR } & \multirow{2}{*}{$\begin{array}{c}\text { FNR } \\
\text { E. coli }\end{array}$} \\
\hline & & P. aeruginosa $\dagger$ & E. coli† & \\
\hline pME3781 & GCTATTGATGTGGATCAACATT & 100 & 100 & 100 \\
\hline pME3773 & AAAT-----A-AT-----AT-- & 40 & 66 & 98 \\
\hline
\end{tabular}

* $\beta$-Galactosidase activities were determined in oxygen-limited cells (Tables 2 and 3) and set at $100 \%$.

† The Anr ${ }^{+}$hosts were the wild-type $P$. aeruginosa PAO1 and the E. colifnr mutant JRG1728 carrying the anr gene of $P$. aeruginosa on pME3777. $\ddagger$ The Fnr ${ }^{+}$host was E. coli MC1000.

(Table 3), as expected from previous mutant studies in $E$. coli (Jayaraman et al., 1989; Bell et al., 1990). The Arc box and the ATCAG half-site mutant gave reduced anaerobic expression in E. coli (efficiencies 41-44\%; Table 3). This decrease was similar, albeit stronger, in $P$. aeruginosa (efficiencies 31-33\%; Table 2). Finally, the symmetrical CTGAT .... ATCAG sequence was poorly recognized by FNR in both $E$. coli and $P$. aeruginosa, confirming earlier results of Lodge et al. (1990) and Galimand et al. (1991). In conclusion, this symmetrical sequence reveals a major difference in recognition specificity between FNR and ANR. In general, the $\beta$-galactosidase levels measured in $E$. coli (Table 3 ) were lower than those in $P$. aeruginosa (Tables 1 and 2). This might be a consequence of the copy numbers of the IncQ vector used, estimated to be about
15 in $E$. coli and thus about half of that found in $P$. aeruginosa (Jeenes et al., 1986).

\section{A 22 bp extended consensus FNR site reduces ANR- dependent anaerobic activation}

An extended, fully symmetrical consensus FNR site (Bell et al., 1989) was constructed in plasmid pME3773 (Fig. 1) and compared to the FNR site used above in plasmid pME3781 where the TTGAT .... ATCAA motif was flanked by nucleotides derived from the arc promoter (Table 4). In conjunction with the -10 sequence TATAAT, FNR activated both types of promoters similarly in E. coli. By contrast, ANR clearly preferred the FNR site flanked by the arc nucleotides over the fully 
symmetrical FNR site, in both $E$. coli and $P$. aeruginosa (Table 4).

\section{DISCUSSION}

\section{ANR and FNR can functionally replace each other under certain conditions}

The cloned anr gene restores anaerobic growth to an $E$. coli fnr mutant on nitrate (Galimand et al., 1991; Sawers, $1991)$ and, reciprocally, the cloned fnr gene complements a $P$. aeruginosa anr mutant for denitrification, as we have shown here. This implies that FNR and ANR can interact with the RNA polymerases of both $E$. coli and $P$. aeruginosa, provided that the promoters satisfy certain requirements; for instance, promoters which contain the consensus FNR site and the $E$. coli consensus -10 sequence meet these requirements. Neither E. coli (Sharrocks et al., 1991; Walker \& DeMoss, 1992; Sawers, 1993; Busby \& Ebright, 1994) nor P. aeruginosa (Gamper et al., 1991) requires a typical -35 promoter sequence for anaerobic induction by FNR/ANR. This fact does not rule out the possibility that the -35 region may influence the magnitude of FNR/ANR-dependent promoter activation.

The presumed loop around Gly-85 in FNR is essential for activating contacts between FNR and E. coli RNA polymerase (Bell \& Busby, 1994). The 85-loop is strictly conserved in ANR (Spiro, 1994) and may thus facilitate contacts between ANR and RNA polymerase of E. coli. Furthermore, using the artificial FNR $/-10$ consensus promoter we have shown here that FNR can activate RNA polymerase of $P$. aeruginosa in vivo. Other $E$. coli proteins that are known to activate $P$. aeruginosa RNA polymerase include $\operatorname{RpoE}\left(\sigma^{\mathrm{E}}\right)$ and $\lambda$ proteins $c \mathrm{I}$ and $c \mathrm{II}$ (Yu et al., 1995; Gao \& Gussin, 1991).

FNR could functionally complement an anr mutant of $P$. aeruginosa for denitrification. The chromosome region encoding nitrite reductase and nitric oxide reductase of $P$. aeruginosa contains several promoters that could be activated by ANR. For instance, the presumed ANR-binding sites in the nirQ and norC promoters are TTGAC.... ATCAA and TTGAT.... ATCAA, respectively (Arai $e t$ al., 1994, 1995a). These sequences are well recognized by FNR in P. aeruginosa (Table 1). We expect that the control regions involved in the expression of other denitrification functions, e.g. nitrate reductase and nitrous oxide reductase, should also contain -40 regions that can interact with FNR, since the entire denitrification pathway of $P$. aeruginosa is anaerobically inducible (Ye et al., 1995).

\section{Some promoters carrying a mutant FNR-binding site are recognized more efficiently by ANR than by FNR}

To facilitate the comparison of anaerobically inducible promoters in $P$. aeruginosa and $E$. coli, we used a set of modular constructs in a broad-host-range IncQ vector (Fig. 1). Because of the multi-copy nature of the vector, high $\beta$-galactosidase levels were observed in some cases, especially in $P$. aeruginosa. A chromosomal $\operatorname{arc} D-l a c Z$ transcriptional fusion in $P$. aeruginosa PAO6265 gave an
E. $\operatorname{coli}$
FNR 196 TRODIGNYL GLT VETISRLLGRFQ
219
P. aeruginosa
ANR 191
SRNEIGNYL GLA VETVSRVPTRFQ
214
P. stutzeri
Fnra 191
SRABIGNYL GLA VETVSRVPTRFQ

Fig. 2. Sequence alignment of the C-terminal helix-turn-helix motifs of FNR, ANR and FnrA. The FNR residues Glu-209, Ser-212 and Arg-213, which are known to have a significant role in the recognition of the FNR box, are conserved in the two Pseudomonas proteins. The residues shown in bold deviate between the E. coli and the two Pseudomonas proteins (Spiro, 1994).

uninduced (aerobic) value of $0.15 \mathrm{kU}$ and an induced (anaerobic) value of $2.4 \mathrm{kU}$. Strain PAO1 carrying pME3533, which is a comparable multi-copy construct, had uninduced and induced $\beta$-galactosidase levels of $1.8 \mathrm{kU}$ and $23.6 \mathrm{kU}$, respectively (values obtained after subtraction of the PAO6261 blanks; Table 1). Thus, the anaerobic induction factor is slightly higher in the monocopy state than in the multi-copy situation. However, it is unlikely that in the latter situation expression was limited by the amount of the regulator.

The constructs containing the -10 hexamer TATAAT showed that ANR was a stronger activator than FNR at the -40 sequences TTGAC.... ATCAG (Arc box), TTGAT .... ATCAG, and CTGAT ... ATCA G in both $P$. aeruginosa and $E$. coli (Tables 2 and 3 ). The first two constructs show the same patterns of ANR- vs FNRdependent expression in both hosts, within the accuracy of the method used. The construct carrying the -40 element CTGAT .... ATCAG was activated much better by ANR than by FNR in E. coli (Table 3). The same trend was observed in $P$. aeruginosa, but for unknown reasons the difference was less pronounced (Table 2). The structural basis for the differential promoter activation by ANR and FNR might be in the helix-turn-helix motifs of the regulators. The role of the FNR helix-turn-helix motif as a DNA-binding domain is well established (Spiro \& Guest, 1987; Bell et al., 1989; Lazazzera et al., 1993; Spiro, 1994). ANR and the closely related FnrA protein of $P$. stutzeri (Cuypers \& Zumft, 1993) have a helix-turn-helix motif which is very similar to that of FNR (Fig. 2). However, there are several FNR residues that are not conserved in ANR and FnrA (Fig. 2). Further work is needed to show whether these differences account for the differential activation specificities of FNR and ANR. Overall, our impression is that ANR may be less stringent in its DNA binding site specificity than FNR. Poor recognition of the arc promoter by FNR explains why FNR cannot complement an anr mutant of $P$. aeruginosa for induction of the arginine deiminase pathway.

\section{RNA polymerases of $P$. aeruginosa and $E$. coli appear to have different -10 sequence specificities}

The $\sigma^{\mathbf{7 0}}$ factors of E. coli and P. aeruginosa are very similar. Region 2 of $\sigma^{70}$, which is important for the recognition of -10 promoter regions, is $100 \%$ conserved in both microorganisms (Tanaka \& Takahashi, 1991; Ronald et al., 1992). Somewhat unexpectedly, we found that the -10 arc hexamer CCTAAT gave different expression in $P$. 
aeruginosa and E. coli. Good promoter activity in $P$. aeruginosa was contrasted by very low activity in E. coli (Tables 1 and 3). Among 300 E. coli promoters analysed, none has a CCTAAT -10 sequence (Lisser \& Margalit, 1993). In Pseudomonas, a PyPuTAAT consensus -10 sequence has been proposed (Ronald et al., 1992; $\mathrm{Py}=\mathrm{C}$ or $\mathrm{T} ; \mathrm{Pu}=\mathrm{A}$ or $\mathrm{G}$ ). Since this consensus sequence is based on a small number of promoters, its validity is uncertain. Our findings leave us with two interpretations. (i) In $P$. aeruginosa, transcription of anaerobically inducible promoters might involve an alternative $\sigma$ factor. This possibility has not been ruled out but seems unlikely considering the excellent activity of promoters having a $\sigma^{70}$ consensus -10 sequence in $P$. aeruginosa. (ii) It is more plausible to assume that in $P$. aeruginosa, by analogy with the situation in E. coli (Sharrocks et al., 1991; Busby \& Ebright, 1994), the ANR (FNR)-dependent promoters are transcribed by $\sigma^{70} \mathrm{RNA}$ polymerase. If such is the case, the -10 specificities of the $\sigma^{70}$ RNA polymerases from the two organisms may be different. In support of this hypothesis, the in vitro data of Gao \& Gussin (1991) suggest that purified $\sigma^{70} \mathrm{RNA}$ polymerase of $P$. aeruginosa may be less stringent than the E. coli enzyme in promoter recognition. In the context of the arc operon of $P$. aeruginosa, the -10 sequence CCTAAT allows the promoter to be tightly controlled by oxygen limitation. The consensus -10 sequence TATAAT leads to extremely high anaerobic expression, but also elevates the aerobic, uninduced expression.

\section{ACKNOWLEDGEMENTS}

We thank Christoph Kündig (Mikrobiologisches Institut, ETH Zürich) and Heinz Baur (Intron, Kaltbrunn/CH) for synthesizing the oligonucleotides used in this study. We also thank Fiona Carruthers for critically reading the manuscript and SmithKline Beecham for generously supplying carbenicillin. This work was supported in part by a grant from the ETH Zürich, where this project was initiated.

\section{REFERENCES}

Arai, H., Igarashi, Y. \& Kodama, T. (1994). Structure and ANRdependent transcription of the nir genes for denitrification from Pseudomonas aeruginosa. Biosci Biotechnol Biochem 58, 1286-1291.

Arai, H., Igarashi, Y. \& Kodama, T. (1995a). The structural genes for nitric oxide reductase from Pseudomonas aeruginosa. Biochim Biophys Acta 1261, 279-284.

Arai, H., Igarashi, Y. \& Kodama, T. (1995b). Expression of the nir and nor genes for denitrification of Pseudomonas aeruginosa requires a novel CRP/FNR-related transcriptional regulator, DNR, in addition to ANR. FEBS Lett 371, 73-76.

Bagdasarian, M. M., Amann, E., Lurz, R., Ruckert, B. \& Bagdasarian, M. (1983). Activity of the hybrid trp-lac (tac) promoter of Escherichia coli in Pseudomonas putida. Construction of broad-host-range, controlled-expression vectors. Gene 26, 273-282.

Bell, A. \& Busby, S. (1994). Location and orientation of an activating region in the Escherichia coli transcription factor, FNR. Mol Microbiol 11, 383-390.

Bell, A. I., Gaston, K. L., Cole, J. A. \& Busby S. J. W. (1989). Cloning of binding sequences for the Escherichia coli transcription activators, FNR and CRP: location of bases involved in dis- crimination between FNR and CRP. Nucleic Acids Res 17, 3865-3874.

Bell, A. I., Cole, J. A. \& Busby, S. J. W. (1990). Molecular genetic analysis of an FNR-dependent anaerobically inducible Escherichia coli promoter. Mol Microbiol 4, 1753-1763.

Busby, S. \& Ebright, R. H. (1994). Promoter structure, promoter recognition, and transcription activation in prokaryotes. Cell 79, 743-746.

Cuypers, H. \& Zumft, W. G. (1993). Anaerobic control of denitrification in Pseudomonas stutzeri escapes mutagenesis of an fnr-like gene. J Bacteriol 175, 7236-7246.

Del Sal, G., Manfioletti, G. \& Schneider, C. (1988). A one-tube plasmid DNA mini-preparation suitable for sequencing. Nucleic Acids Res 16, 9878.

Dreyfus, M. (1988). What constitutes the signal for the initiation of protein synthesis on Escherichia coli mRNAs? J Mol Biol 204, 79-94.

Eiglmeier, K., Honoré, N., luchi, S., Lin, E. C. C. \& Cole, S. T. (1989). Molecular genetic analysis of FNR-dependent promoters. Mol Microbiol 3, 869-878.

Galimand, M., Gamper, M., Zimmermann, A. \& Haas, D. (1991). Positive FNR-like control of anaerobic arginine degradation and nitrate respiration in Pseudomonas aeruginosa. $J$ Bacteriol 173, 1598-1606.

Gamper, M., Zimmermann, A. \& Haas, D. (1991). Anaerobic regulation of transcription initiation in the $\operatorname{arcD} A B C$ operon of Pseudomonas aeruginosa. J Bacteriol 173, 4742-4750.

Gao, J. \& Gussin, G. N. (1991). RNA polymerases from Pseudomonas aeruginosa and Pseudomonas syringae respond to Escberichia coli activator proteins. J Bacteriol 173, 394-397.

Green, J. \& Guest, J. R. (1994). Regulation of transcription at the $n d h$ promoter of Escherichia coli by FNR and novel factors. Mol Microbiol 12, 433-444.

Green, J., Trageser, M., Six, S., Unden, G. \& Guest, J. R. (1991). Characterization of the FNR protein of Escherichia coli, an ironbinding transcriptional regulator. Proc R Soc Lond B 244, 137-144.

Green, J., Sharrocks, A. D., Green, B., Geisow, M. \& Guest, J. R. (1993). Properties of FNR proteins substituted at each of the five cysteine residues. Mol Microbiol 8, 61-68.

Haas, D., Gamper, M. \& Zimmermann, A. (1992). Anaerobic control in Pseudomonas aeruginosa. In Pseudomonas: Molecular Biology and Biotechnology, pp. 177-187. Edited by E. Galli, S. Silver \& B. Witholt. Washington, DC: American Society for Microbiology.

Jayaraman, P.-S., Cole, J. A. \& Busby S. J. W. (1989). Mutational analysis of the nucleotide sequence at the FNR-dependent nirB promoter in Escherichia coli. Nucleic Acids Res 17, 135-145.

Jeenes, D. J., Soldati, L., Baur, H., Watson, J. M., Mercenier, A., Reimmann, C., Leisinger, T. \& Haas, D. (1986). Expression of biosynthetic genes from Pseudomonas aeruginosa and Escherichia coli in the heterologous host. Mol \& Gen Genet 203, 421-429.

Kahn, M., Kolter, R., Thomas, C., Figurski, D., Meyer, R., Remaut, E. \& Helinski, D. R. (1979). Plasmid cloning vehicles derived from plasmids ColE1, F, R6K, and RK2. Methods Enzymol 68, 268-280.

Khoroshilova, N., Beinert, H. \& Kiley, P. J. (1995). Association of a polynuclear iron-sulfur center with a mutant FNR protein enhances DNA binding. Proc Natl Acad Sci US A 92, 2499-2503.

Lazazzera, B. A., Bates, D. M. \& Kiley, P. J. (1993). The activity of the Escherichia coli transcription factor FNR is regulated by a change in oligomeric state. Genes \& Dev 7, 1993-2005.

Lisser, S. \& Margalit, H. (1993). Compilation of E. coli mRNA promoter sequences. Nucleic Acids Res 21, 1507-1516.

Lodge, J., Williams, R., Bell, A., Chan, B. \& Busby, S. (1990). 
Comparison of promoter activities in Escherichia coli and Pseudomonas aeruginosa: use of a new broad-host-range promoter-probe plasmid. FEMS Microbiol Lett 67, 221-226.

Luthi, E., Mercenier, A. \& Haas, D. (1986). The $\operatorname{arc} A B C$ operon required for fermentative growth of Pseudomonas aeruginosa on arginine: Tn5-751-assisted cloning and localization of structural genes. J Gen Microbiol 132, 2667-2675.

Luthi, E., Baur, H., Gamper, M., Brunner, F., Villeval, D., Mercenier, A. \& Haas, D. (1990). The arc operon for anaerobic arginine catabolism in Pseudomonas aeruginosa contains an additional gene, $\operatorname{arcD}$, encoding a membrane protein. Gene 87, 37-43.

Minton, N. P. (1984). Improved plasmid vectors for the isolation of translational lac gene fusions. Gene 31, 269-273.

Ronald, S., Farinha, M. A., Allan, B. J. \& Kropinski, A. M. (1992). Cloning and physical mapping of transcriptional regulatory (sigma) factors from Pseudomonas aeruginosa. In Pseudomonas: Molecular Biology and Biotechnology, pp. 249-257. Edited by E. Galli, S. Silver \& B. Witholt. Washington, DC: American Society for Microbiology.

Sambrook, J., Fritsch, E. F. \& Maniatis, T. (1989). Molecular Cloning: a Laboratory Manual, 2nd edn. Cold Spring Harbor, NY: Cold Spring Harbor Laboratory.

Savioz, A., Zimmermann, A. \& Haas, D. (1993). Pseudomonas aeruginosa promoters which contain a conserved GG-N ${ }_{10}-G C$ motif but appear to be RpoN-independent. Mol $\mathcal{E}$ Gen Genet 238, 74-80.

Sawers, R. G. (1991). Identification and molecular characterization of a transcriptional regulator from Pseudomonas aeruginosa PAO1 exhibiting structural and functional similarity to the FNR protein of Eschericbia coli. Mol Microbiol 5, 1469-1481.

Sawers, G. (1993). Specific transcriptional requirements for positive regulation of the anaerobically inducible $p f$ operon by ArcA and FNR. Mol Microbiol 10, 737-747.

Sharrocks, A. D., Green, J. \& Guest, J. R. (1991). FNR activates and represses transcription in vitro. Proc $R$ Soc Lond B 245, 219-226.

Shaw, D. J. \& Guest, J. R. (1982). Nucleotide sequence of the $f n r$ gene and primary structure of the Fnr protein of Escherichia coli. Nucleic Acids Res 10, 6119-6130.

Spiro, S. (1994). The FNR family of transcriptional regulators. Antonie Leeuwenboek 66, 23-36.
Spiro, S. \& Guest, J. R. (1987). Activation of the lac operon of Escherichia coli by a mutant FNR protein. Mol Microbiol 1, 53-58.

Spiro, S. \& Guest, J. R. (1990). FNR and its role in oxygen-regulated gene expression in Escberichia coli. FEMS Microbiol Rev 75, 399-428.

Spiro, S., Gaston, K. L., Bell, A. I., Roberts, R. E., Busby, S. J. W. \& Guest, J. R. (1990). Interconversion of the DNA-binding specificities of two related transcription regulators, CRP and FNR. Mol Microbiol 4, 1831-1838.

Tanaka, K. \& Takahashi, H. (1991). Cloning and analysis of the gene $r p o D A$ for the principal $\sigma$ factor of Pseudomonas aeruginosa. Biochim Biophys Acta 1089, 113-119.

Unden, G., Becker, S., Bongaerts, J., Holighaus, G., Schirawski, J. \& Six, S. (1995). $\mathrm{O}_{2}$-sensing and $\mathrm{O}_{2}$-dependent gene regulation in facultatively anaerobic bacteria. Arch Microbiol 164, 81-90.

Walker, M. S. \& DeMoss, J. A. (1991). Promoter sequence requirements for Fnr-dependent activation of transcription of the narGHJI operon. Mol Microbiol 5, 353-360.

Walker, M. S. \& DeMoss, J. A. (1992). Role of alternative promoter elements in transcription from the nar promoter of Escherichia coli. $J$ Bacteriol 174, 1119-1123.

Ye, R. W., Haas, D., Ka, J.-O., Krishnapillai, V., Zimmermann, A., Baird, C. \& Tiedje, J. M. (1995). Anaerobic activation of the entire denitrification pathway in Pseudomonas aeruginosa requires Anr, an analog of Fnr. J Bacteriol 177, 3606-3609.

Yu, H., Schurr, M. J. \& Deretic, V. (1995). Functional equivalence of Escherichia coli $\sigma^{\mathbf{E}}$ and Pseudomonas aeruginosa AlgU: E. coli rpoE restores mucoidy and reduces sensitivity to reactive oxygen intermediates in alg $U$ mutants of $P$. aeruginosa. $J$ Bacteriol 177, 3259-3268.

Ziegelhoffer, E. C. \& Kiley P. J. (1995). In vitro analysis of a constitutively active mutant form of the Escherichia coli global transcription factor FNR. $J$ Mol Biol 245, 351-361.

Zimmermann, A., Reimmann, C., Galimand, M. \& Haas, D. (1991). Anaerobic growth and cyanide synthesis of Pseudomonas aeruginosa depend on anr, a regulatory gene homologous with fnr of Escherichia coli. Mol Microbiol 5, 1483-1490.

Received 17 August 1995; revised 23 October 1995; accepted 2 November 1995. 